\title{
An investigation of lingual vibrotactile detectability
}

\author{
KAL M. TELAGE and PETER J. GOLEMAN \\ Ithaca College, Ithaca, New York
}

\begin{abstract}
The purpose of the study was to investigate lingual detectability of vibrotactile stimuli presented at different frequencies. Detectability was assessed objectively in comparing data expressed in millivolts and microns of peak contractor displacements. Detectability was also measured subjectively in terms of individual ratings of how clearly the stimulus was perceived throughout threshold determination. An interesting finding was that the frequency resulting in the most sensitive thresholds $(250 \mathrm{~Hz})$ was the least detectable and showed the greatest threshold variability across subjects. The authors argue for developing clinical testing paradigms that take full advantage of stimulus detectability over frequency sensitivity, which has been previously emphasized.
\end{abstract}

The primary focus of oral vibrotactile research during the last 10 years has been methodological in nature. It has attempted to improve the instrumentation (Fucci \& Kelly, 1972; Telage, 1979), develop and facilitate alternate measurement procedures (Telage \& Fucci, 1974; Fucci, Curtis, \& McCaffrey, 1975; Telage, Fucci, \& Blackmon, 1976; Telage \& Warren, 1977), and identify the nature and function of oral lingual tactile transducers that mediate sensory control during speech production (Telage \& Petrosino, 1978; Telage \& Scott, 1980). A major goal of the methodologic research has been to develop a diagnostic instrument for assessing the functional integrity of the tactile feedback mechanism. The basic interest in this research stems from a series of closed-loop models of the speech-production process that emphasize the importance of haptic sensory feedback modalities in the control of patterned motor speech behaviors. The present investigation is concerned with facilitating measurement procedures by obtaining both subjective and objective data relative to lingual detectability of the vibrotactile stimulus at selected frequencies of vibration.

\section{METHOD}

\section{Subjects}

Eight normal adults between the ages of 19 years 11 months and 27 years 2 months participated. Each of the subjects was trained to respond to lingual vibrotactile stimuli during a 15 -min preexperimental session. The training session included explaining the testing process, indicating the manner of responding to the sensory stimuli, and obtaining six vibrotactile thresholds from the anterior midline surface of the tongue (a frequency of $250 \mathrm{~Hz}$ was used). To ensure adequacy of training for all subjects, a selection criterion required three of six thresholds obtained to differ by no more than $10 \mathrm{mV}$ of stimulus intensity. All of the subjects met the selection criterion subsequent to their training session.

\section{Apparatus}

Figure 1 presents a block diagram of the instrument package used in this study. The stimulus control unit is composed of Coulbourn solid-

The authors' mailing address is: School of Allied Health Professions, Speech Pathology-Audiology, Ithaca College, Ithaca, NY 14850.

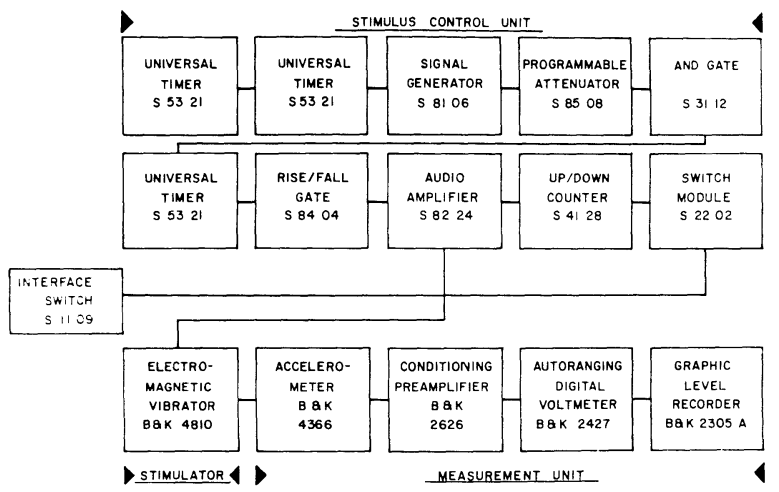

Figure 1. A block diagram of the instrumentation package used in the experiment.

state logic modules. These units generate pulsed vibratory signals that may be varied in frequency, intensity, and temporal characteristics. Three universal timers are programmed to control signal duration and duty cycle. The timers gate a selectable rise-fall module on and off. This continuously adjustable electronic switch is set to generate a rise-fall time of $100 \mathrm{msec}$. The signal from the rise-fall module is fed into a precision signal generator and an audio mixer amplifier. Stimulus intensities are varied in increments of 256.5 -dB steps by passing them through a programmable attenuator and an 8-bit binary up-down counter. Pulsed signals from the stimulus control unit drive the electromagnetic minivibrator that is the stimulus-producing aggregate of the system.

\section{Procedure}

Figure 2 presents a schematic diagram of the vibrator and free-surround disk. During threshold measurements, each subject reclined in a motorized dental lounge adjusted for easy access to the vibrator apparatus. With the head reclined and supported, the lounge was elevated to allow subjects to press the anterior midline surface of the tongue against the surround disk. While the subject was in this position, the contactor $\left(.32 \mathrm{~cm}^{2}\right)$ was lowered through the opening in the disk until it extended $1 \mathrm{~mm}$ into the lingual surface. The diameter of the opening in the upper surround disk was $2 \mathrm{~mm}$ larger than the diameter of the contactor.

Three lingual vibrotactile thresholds were obtained from each subject at stimulus frequencies of $30,50,100$, and $250 \mathrm{~Hz}$; counterbalanced orders of presentation were used. To eliminate any fatigue or adaptation effects, rest periods of 2 min between threshold trials and 3 min between frequency presentations were used. 


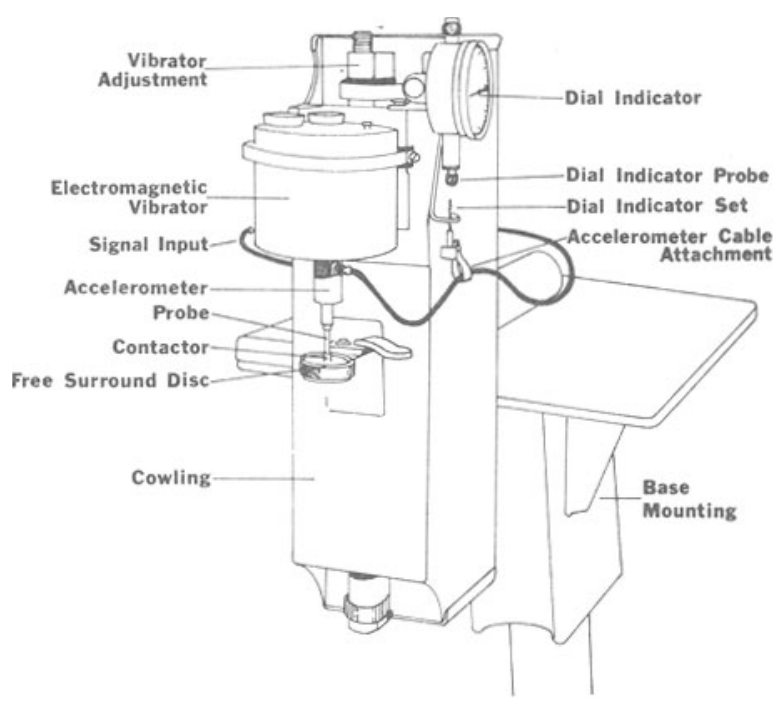

Figure 2. Schematic diagram of the free-surround disk and vibrator portions of the oral vibrotactile stimulator.

Thresholds were determined by a psychophysical method of limits. Pulsed stimuli (on $1.5 \mathrm{sec}$, off $1.0 \mathrm{sec}$ ) were initially presented at $100 \mathrm{mV}$ of contactor displacement and were automatically attenuated in steps of approximately $5 \mathrm{mV}$ pulse. A wide-band noise generator presented auditory masking at $90 \mathrm{~dB}$ SPL sound field to eliminate auditory stimulus cues. The subject pressed an interface switch when they could no longer detect the vibratory stimulus.

An accelerometer, mounted on the probe, and set to emit $100 \mathrm{mV} / \mathrm{g}$ of acceleration, continuously measured contactor displacement as a small voltage. Displacement voltages were then directly amplified by a conditioning preamplifier and monitored by a digital voltmeter set to read peak displacement values in millivolts. The median of three threshold trials was accepted as threshold. A formula for acceleration conversion (Telage, 1979) converted data initially recorded in millivolts to microns of peak displacement.

At the conclusion of each series of three threshold trials, the subjects were required to rate subjectively the physical detectability of each of the four test frequencies used. A 4-point rating scale ( $1=$ most difficult to detect) was employed.

\section{RESULTS AND DISCUSSION}

Table 1 presents means and standard deviations for vibrotactile thresholds expressed in millivolts of peak contactor displacement. These data are shown in millivolts to indicate that it takes greater energy to obtain lingual thresholds as vibratory frequency increases. In determining audiometric thresholds, for example, the energy requirements for the intensity settings across the frequency

Table 1

Means and Standard Deviations for Lingual Vibrotactile Thresholds Expressed in Millivolts of Peak Displacement $(\mathbf{N}=\mathbf{8})$

\begin{tabular}{ccccc}
\hline & \multicolumn{4}{c}{ Threshold Values } \\
\cline { 2 - 5 } & $30 \mathrm{~Hz}$ & $50 \mathrm{~Hz}$ & $100 \mathrm{~Hz}$ & $250 \mathrm{~Hz}$ \\
\hline Mean & 4.4 & 4.8 & 9.1 & 38.9 \\
SD & 1.1 & 1.2 & 6.0 & 11.9 \\
\hline
\end{tabular}

range are built into the instrumentation. The relative differences in energy are not apparent. In vibrotactile measurements, the differential energy requirements across frequency are inherent in the thresholds themselves and are readily apparent when data are expressed in millivolts. This is observed clearly in the threshold pattern in Table 1 . The $30-$ to- $50-\mathrm{Hz}$ range requires an average of 4.4 and $4.8 \mathrm{mV}$ for threshold detectability. At $100 \mathrm{~Hz}$, the energy requirement nearly doubles. At $250 \mathrm{~Hz}$, the energy requirement is nearly nine times greater than that at 30 $\mathrm{Hz}$. Standard deviations indicate a greater consistency in threshold determinations by subjects at the two lowest frequencies.

It is important to point out that the data in Table 1 do not reflect lingual sensitivity to the vibrotactile stimulus. Although the millivolt data indicate the energy needed to detect the signal at threshold at the selected frequencies, millivolt data do not indicate the amplitude of peak displacement of the vibrating contactor. To observe thresholds as a function of lingual sensitivity, the following formula is used to convert data to microns of peak displacement:

$$
\begin{aligned}
\text { Microns } & =\mu \mathrm{V} \times 250,000 \\
& =\mathrm{F}^{\mathrm{z}} \times(\text { sensitivity of accelerometer in } \mu \mathrm{V} / \mathrm{g}) .
\end{aligned}
$$

Table 2 presents means and standard deviations for vibrotactile threshold converted to microns of peak contactor displacement. In comparing Tables 1 and 2, note the inverse relationship between energy in millivolts and displacement in microns as frequency increases. At slower vibratory cycles, small amounts of energy produce considerably greater amplitudes of peak displacement of the contactor into the lingual surface than are produced at higher oscillations. This is clearly reflected in the conversion formula, in which the frequency of vibration is squared and multiplied by a constant. Increasing the frequency results in larger denominators and relatively smaller micron values. The data in Table 2 show a pattern of increasing threshold sensitivity up to $250 \mathrm{~Hz}$. When the frequency range is increased above $250 \mathrm{~Hz}$, a typical U-shaped curve emerges. These data are consistent with previous vibrotactile studies that have shown maximum threshold sensitivity at $250 \mathrm{~Hz}$ (Geldard, 1972).

Clearly, data in this study, as well as in previous research, indicate that $250 \mathrm{~Hz}$ is the most sensitive frequency in the range used for oral vibrotactile threshold measurements. "Sensitivity" refers to subjects' being

Table 2

\begin{tabular}{ccccc}
$\begin{array}{c}\text { Means and Standard Deviations for Lingual Vibrotactile } \\
\text { Thresholds Expressed in Microns } \\
\text { of Peak Displacement } \mathbf{( N = 8 )}\end{array}$ \\
\cline { 2 - 5 } & \multicolumn{4}{c}{ Threshold Values } \\
\hline & $30 \mathrm{~Hz}$ & $50 \mathrm{~Hz}$ & $100 \mathrm{~Hz}$ & $250 \mathrm{~Hz}$ \\
\hline Mean & 24.6 & 9.6 & 4.5 & 3.1 \\
SD & 2.4 & 2.3 & 3.0 & 1.3 \\
\hline
\end{tabular}


Table 3

Individual Subjective Ratings, and Means and Standard Deviations, for Stimulus Detectability for each Frequency Tested $(\mathbf{N}=\mathbf{8})$

Subjective Ratings of Stimulus Detectability

\begin{tabular}{ccccc}
\cline { 2 - 5 } Subject & $30 \mathrm{~Hz}$ & $50 \mathrm{~Hz}$ & $100 \mathrm{~Hz}$ & $250 \mathrm{~Hz}$ \\
\hline 1 & 3 & 2 & 1 & 4 \\
2 & 3 & 2 & 1 & 4 \\
3 & 2 & 1 & 3 & 4 \\
4 & 1 & 3 & 2 & 4 \\
5 & 1 & 2 & 3 & 4 \\
6 & 1 & 3 & 2 & 4 \\
7 & 2 & 1 & 3 & 4 \\
8 & 1 & 2 & 3 & 4 \\
Mean & $1-7$ & $2-0$ & $2-2$ & 4 \\
SD & $0-8$ & $0-7$ & $0-8$ & 0 \\
\hline
\end{tabular}

aware of the stimulus with the least amount of contactor displacement into the tongue. Since highly sensitive frequencies are often used for vibrotactile threshold measurements, it is possible that some experimenters have equated frequency sensitivity with lingual vibrotactile detectability. It does not follow, however, that sensitive frequencies, that is, those that produce the lowest thresholds, are highly detectable frequencies to use for clinical testing. In fact, clinical subjects have shown some variability in their respective abilities to continue to track a stimulus at near-threshold levels. Both the high vibratory rate $(250$ pulses/sec) and the high sensitivity (some awareness is apparent at displacements as small as 3.14 microns) may be contributing to the ability of subjects to provide the most reliable threshold data. Standard deviations for both the millivolt and micron data indicate greater intersubject threshold consistency at 30 and $50 \mathrm{~Hz}$ than at the higher frequencies.

Table 3 presents subjective detectability ratings for the frequencies used to obtain thresholds. The ratings are based on a 4-point scale in which each number could be used only once. It is clear that the three lowest frequencies were rated most detectable. Of even greater significance is the fact that all subjects rated $250 \mathrm{~Hz}$ as the least detectable of the frequencies used in this investigation. It appears that the higher rate of vibration is more difficult to track at low intensities and influences the reliability of threshold determinations. The nature of clinical research, particularly comparative clinical studies, argues for developing testing paradigms that take full advantage of stimulus detectability over frequency sensitivity. Only in this manner can clinically significant differences between normal and abnormal lingual sensory functions be observed.

\section{REFERENCES}

FuCCI, D., CurTis, A. P., \& McCaffrey, P. (1975). Lingual vibrotactile threshold alterations in response to varying stimulus levels of intensity and duration. Perceptual and Motor Skills, 41, 127-133.

FuCCI, D., \& KeLLY, D. (1972). New instrumentation for research on vibrotactile sensitivity of the tongue. Review of Scientific Instruments, 43, 1748-1751.

Geldard, F. (1972). The human senses. Wiley.

Telage, K. (1979). Automated instrumentation for research on the lingual tactile sensory system. Review of Scientific Instruments, 50, 1296-1298.

Telage, K., \& FucCI, D. (1974). Concerning intrasubject measurements of successive lingual vibrotactile responses. Perceptual and Motor Skills, 39, 1047-1052.

Telage, K., Fucci, D., \& Blackmon R. (1976). Temporal summation effects on lingual vibrotactile thresholds. Perceptual and Motor Skills, 42, 859-864.

Telage, K., \& Petrosino, L. (1978). Testing procedures for measuring oral vibrotactile thresholds: III. Effects obtained using a nonclamping method. Bulletin of the Psychonomic Society, 12, 291-293.

TelaGE, K., \& ScoTt, J. C. (1980). Effects of auditory interference upon observed lingual tactile thresholds. Bulletin of the Psychonomic Society, 15, 422-424.

Telage, K., \& WarRen, J. (1977). Spatial summation effects on lingual vibrotactile thresholds. Perceptual and Motor Skills, 44, 1179-1185.

(Manuscript received for publication July 27, 1984.) 